\title{
Lattice study of transport coefficients in second order dissipative hydrodynamics
}

\author{
Yasuhiro Kohno* \\ Department of Physics, Osaka University, Toyonaka 560-0043, Japan \\ E-mail: kouno@kern.phys.sci.osaka-u.ac.jp
}

\section{Masayuki Asakawa}

Department of Physics, Osaka University, Toyonaka 560-0043, Japan

E-mail: yuki@phys.sci.osaka-u.ac.jp

\section{Masakiyo Kitazawa}

Department of Physics, Osaka University, Toyonaka 560-0043, Japan

E-mail: kitazawa@phys.sci.osaka-u.ac.jp

\section{Chiho Nonaka}

Kobayashi-Maskawa Institute for the Origin of Particles and the Universe (KMI),

Department of Physics, Nagoya University, Furo-cho, Chikusa-ku, Nagoya 464-8602, Japan

E-mail: nonaka@hken.phys.nagoya-u.ac.jp

\begin{abstract}
The quark-gluon plasma produced in heavy ion collisions at RHIC energy is known to be very close to the ideal fluid. Calculations with viscosities and other transport coefficients, i.e. second order dissipative hydrodynamics, are on the way. While second order dissipative hydrodynamics is known as a causal theory, it includes many phenomenological transport coefficients. We attempt to constrain those transport coefficients by SU(3) lattice gauge calculation. On the basis of the phenomenological derivation of second order hydrodynamics by Israel-Stewart and Einstein principle, we relate the Israel-Stewart parameters to fluctuations of off-diagonal components of energy-momentum tensor on the lattice and evaluate them.
\end{abstract}

The XXVIII International Symposium on Lattice Field Theory, Lattice2010

June 14-19, 2010

Villasimius, Italy

\footnotetext{
${ }^{*}$ Speaker.
} 


\section{Introduction}

One of the striking features of quark-gluon plasma (QGP) produced at RHIC (the Relativistic Heavy Ion Collider) is the fact that the space-time evolution of the matter produced in heavy ion collisions is well described by hydrodynamic models [1]. Heavy ion collisions which will be started at the LHC (the Large Hadron Collider) can reach higher temperature $(T)$ than RHIC. The value of shear viscosity, $\eta$, is then expected to increase, since the strong coupling constant becomes smaller because of the aymptotic freedom of Quantum Chromodynamics (QCD). Indeed, leading-order perturbative calculation predicts the dependences of $\eta$ on $T$ and coupling $g$ as

$$
\eta=\kappa \frac{T^{3}}{g^{4} \ln g^{-1}}
$$

with a positive coefficient $\kappa$ [胞]. It is therefore essential to take into account the effects of disspations in hydrodynamic models for heavy ion collisions at LHC energy.

The simplest relativistic dissipative hydrodynamics is the first order theory, which is proposed by Eckart and Landau-Lifshitz [3, 4]. The first order theory, however, accompanies acausal problem and instability in numerical simulations [5]. A strategy to evade these problems is to extend the theory to second order. One of the hydrodynamic equations given along this line is the Israel-Stewart (IS) theory [6]. In the second order theories, however, there appear many phenomenological parameters that are not included in the first order one. These new parameters cannot be determined within hydrodynamics but should be determined by microscopic theory, i.e. QCD in our case. To constrain the parameters in the hydrodynamic models, microscopic analyses of the transport coefficients are highly desirable.

Since the temperature region realized at RHIC or LHC is not within the reach of perturbation theory, one needs nonperturbative approaches to determine the transport coefficients. At present, lattice gauge theory is only the systematic approach that can calculate physical quantities in such a nonperturbative region. In the present study, we attempt to constrain the phenomenological parameters in the second order hydrodynamics on the lattice using a method proposed in Refs. [0, 8, 2]. In this method, ratios between the viscosities and the relaxation times in the second order theory are related to static fluctuations of energy-momentum tensor in equilibrium. An advantage of this method is that hydrodynamic parameters are constrained only through the measurements of static observables on the lattice. On the other hand, measurements of viscosity using Kubo formulas require the analytic continuation to extract the real-time spectral function from Euclidean correlator. The main purpose of the present work is to evaluate these ratios with $\mathrm{SU}(3)$ gauge theory on the lattice and reduce the number of phenomonological parameters in IS theory.

\section{Second order transport coefficients}

In the following, we first briefly review IS theory, which is a second order dissipative hydrodynamics [6, 10]. Basic equations of hydrodynamics are the local conservation laws of the energy-momentum and the net charge,

$$
\begin{aligned}
& \partial_{\mu} T^{\mu v}=0, \\
& \partial_{\mu} N^{\mu}=0
\end{aligned}
$$


where $T^{\mu v}=T_{\mathrm{eq}}^{\mu v}+\delta T^{\mu v}$ and $N^{\mu}=N_{\mathrm{eq}}^{\mu}+\delta N^{\mu}$ are the energy-momentum tensor and the charge current in nonequilibrium, respectively. Here, $T_{\mathrm{eq}}^{\mu v}$ and $N_{\mathrm{eq}}^{\mu}$ denote the equilibirum values, and $\delta T^{\mu v}$ and $\delta N^{\mu}$ represent the effects of dissipations.

In Eqs. (2.1) and (2.2), there exist fourteen unknown variables for five constituent equations. These equations therefore are not closed. The simplest prescription to introduce additional nine constraints is the ideal fluid approximation, where the number of unknown variables is reduced by neglecting all dissipative effects. Another method is to constrain the system by the second law of thermodynamics,

$$
\partial_{\mu} s^{\mu} \geq 0
$$

This constraint, together with the lineality of dissipative terms against the thermodynamic forces, provides nine constituent equations, which make the hydrodynamic equations closed. Here, the entropy current $s^{\mu}$ is given by

$$
s^{\mu}=s_{\mathrm{eq}} u^{\mu}+\frac{q^{\mu}}{T}+Q^{\mu}
$$

where $s_{\mathrm{eq}}, u^{\mu}$ and $q^{\mu}$ are the entropy density in equilibrium, arbitrary 4-velocity of the fluid normalized as $u_{\mu} u^{\mu}=1$, and the heat flow, respectively. $Q^{\mu}$ represents higher order in dissipative terms, $\delta T^{\mu \nu}$ and $\delta N^{\mu}$. If we neglect $Q^{\mu}$, the second law of thermodynamics and linearity lead to the first order hydrodynamic equations including three transport coefficients, shear and bulk viscosities $\eta$ and $\zeta$, and heat conductivity $\lambda$. The first order equations, however, are known to violate causality.

In the second order theory, one incorporates $Q^{\mu}$ and repeats the similar argument. Up to second order in $\delta T^{\mu v}$ and $\delta N^{\mu}$, the most general form of $Q^{\mu}$ is given by

$$
Q^{\mu}=-\frac{u^{\mu}}{2 T}\left(\beta_{0} \Pi^{2}-\beta_{1} q_{v} q^{v}+\beta_{2} \pi_{\rho \lambda} \pi^{\rho \lambda}\right)-\frac{\alpha_{0} \Pi q^{\mu}}{T}+\frac{\alpha_{1} \pi^{\mu v} q_{v}}{T}+R^{\mu}
$$

where $\Pi$ and $\pi^{\mu v}$ are dissipative currents corresponding to bulk and shear flows, respectively, included in $\delta T^{\mu \nu}$ and $\delta N^{\mu} . R^{\mu}$ represents terms needed for the theory with the conserved currents $N^{\mu}$. Here, we note that dissipative currents are regarded as state variables describing the macroscopic system in dissipative hydrodynamics; accordingly entropy current should be extended as a function of these variables, $s^{\mu}=s^{\mu}\left(\Pi, q^{\mu}, \pi^{\mu v}\right)$. The proportional coefficients $\beta_{i}$ and $\alpha_{i}$ are not determined in this treatment and constitute new phenomenological parameters in IS theory. Eq. (2.5) and the second law of thermodynamics lead to a set of closed equations, i.e. IS equations [6].

A remark is in order. In the above argument we constructed the second order hydrodynamics starting from the entropy current Eq. (2.4) and second law of thermodynamics. The higher-order hydrodynamics equations are also derived by the derivative expansions of $u^{\mu}$ [11]. In the second order theory in the derivative expansion, one can obtain the form of Eq. (2.4) as an extension of the entropy current to nonequilibrium states, which is a monotonically increasing function with small dissipations [11].

One of the IS equations reads

$$
\tau_{\pi} D \pi_{\mu v}=-\pi_{\mu v}+2 \eta \sigma_{\mu v}-\pi_{\mu \nu} \eta T \partial_{\rho}\left(\frac{\tau_{\pi} u^{\rho}}{2 \eta T}\right)+2 \eta \alpha_{1} \nabla_{\langle\mu} q_{v\rangle}
$$


where $D \equiv u_{\mu} \partial^{\mu}$ being the derivative along $u^{\mu}, \sigma_{\mu v} \equiv \frac{1}{2} \Delta_{\mu}^{\alpha} \Delta_{v}^{\beta}\left(\partial_{\alpha} u_{\beta}+\partial_{\beta} u_{\alpha}\right)-\frac{1}{3} \Delta_{\mu v} \partial_{\rho} u^{\rho}$ is the shear tensor, and $\Delta^{\mu v} \equiv g^{\mu v}-u^{\mu} u^{v}$ is the spatial projection tensor orthogonal to $u^{\mu}$, respectively. The angular bracket notation is defined by $A^{\langle\mu v\rangle} \equiv\left[\frac{1}{2}\left(\Delta_{\rho}^{\mu} \Delta_{\sigma}^{v}+\Delta_{\sigma}^{\mu} \Delta_{\rho}^{v}-\frac{1}{3} \Delta^{\mu v} \Delta_{\rho \sigma}\right)\right] A^{\rho \sigma}$. The left hand side of Eq. (2.6) gives rise to relaxation effect to the solution of the first order equations, and the time scale of this relaxation, $\tau_{\pi}$, is called the relaxation time for shear channel. The violation of causality in the first order theory is cured with this term with sufficiently large $\tau_{\pi}$. Although not shown, the IS equation for the bulk channel also has a similar term describing the relaxation with relaxation time $\tau_{\Pi}$. Relaxation times $\tau_{\pi}$ and $\tau_{\Pi}$ are related to $\beta_{i}$ in Eq. (2.5) as

$$
\tau_{\Pi}=\beta_{0} \zeta, \quad \tau_{\pi}=2 \beta_{2} \eta
$$

Eq. (2.7) implies that the ratios between viscosities and the relaxation times are related to proportional coefficients in the entropy current, $\beta_{0}$ and $\beta_{2}$, as

$$
\beta_{0}=\frac{\tau_{\Pi}}{\zeta}, \quad \beta_{2}=\frac{\tau_{\pi}}{2 \eta}
$$

\section{Fluctuations of energy-momentum tensor}

Equations (2.8) show that the measurement of $\beta_{0}$ and $\beta_{2}$ enables us to determine the relaxation time to viscosity ratios. In the following we relate $\beta_{i}$ to fluctuations of energy-momentum tensor using the Einstein principle in order to measure them on the lattice.

Let us first recapitulate the Einstein principle. When the system posseses a set of state variables $\vec{x}=\left(x_{1}, x_{2}, x_{3}, \ldots\right)$, the entropy in equilibrium is given by the function of these variables, $S(\vec{x})$. The entropy then is related to the number of microscopic states corresponding to $\vec{x}, W(\vec{x})$, by Boltzmann principle,

$$
S(\vec{x})=\log W(\vec{x}) .
$$

Einstein rewrote this principle as follows [12],

$$
W(\vec{x})=\exp [S(\vec{x})]
$$

Then, a microscopic state for a set of state variables $\vec{x}$ occurs with the probability

$$
P(\vec{x})=\frac{W(\vec{x})}{\sum W(\vec{x})} .
$$

Following IS theory, the entropy in unit volume $s$ in the rest frame is given by

$$
s\left(\Pi, q^{\mu}, \pi^{\mu v}\right)=u_{\mu} s^{\mu}=s_{\mathrm{eq}}-\frac{1}{2 T}\left(\beta_{0} \Pi^{2}-\beta_{1} q_{\mu} q^{\mu}+\beta_{2} \pi_{\mu v} \pi^{\mu v}\right),
$$

where orthogonality relations $u_{\mu} q^{\mu}=u_{\mu} \pi^{\mu v}=0$ have been used. Substituting Eq. (3.4) into Eq. (3.2), the probability that $\Pi, q^{\mu}$, and $\pi^{\mu v}$ are macroscopically realized in a volume $V$ is given by

$$
P\left(\Pi, q^{\mu}, \pi^{\mu v}\right) \propto \exp \left[-\frac{V}{2 T}\left(\beta_{0} \Pi^{2}-\beta_{1} q_{\mu} q^{\mu}+\beta_{2} \pi_{\mu v} \pi^{\mu v}\right)\right]
$$


where $\Pi, q^{\mu}$, and $\pi^{\mu v}$ are spatial average of corresponding dissipative terms in a volume $V$, defined, for example, as

$$
\pi_{\mu v}=\frac{1}{V} \int_{V} d^{3} x \pi_{\mu v}(x)
$$

The probability distribution of $\pi^{\mu v}$ is then, for example, given by

$$
P\left(\pi_{\mu v}\right) \propto \exp \left[-\frac{V}{2 T} \beta_{2} \pi_{\mu v}^{2}\right],
$$

which leads to the variance for $\pi_{\mu v}$

$$
\left\langle\left(V \pi_{\mu v}\right)^{2}\right\rangle=\frac{V T}{\beta_{2}}
$$

where we have used $\left\langle\pi^{\mu v}\right\rangle=0$. Since in the rest frame one has $\pi_{i j}=T_{i j}=\frac{1}{V} \int_{V} d^{3} x T_{i j}(x)$ for $1 \leq i<j \leq 3$, with Eq. (3.8) one obtains the formula for $\beta_{2}$ in the rest frame as

$$
\beta_{2}=\frac{T}{V\left\langle T_{i j}^{2}\right\rangle} .
$$

Finally, we remark that Eq. (3.9) is also obtained via the relaxation-time approximation for the correlation function of the energy-momentum tensor [8]

$$
\left\langle\pi_{i j}(t) \pi_{i j}(0)\right\rangle=\left\langle\pi_{i j}(0) \pi_{i j}(0)\right\rangle \mathrm{e}^{-t / \tau_{\pi}} .
$$

The fluctuation of the energy-momentum tensor $\left\langle T_{\mu v}^{2}\right\rangle$ is rewritten as

$$
\left\langle T_{\mu v}^{2}\right\rangle=\frac{1}{V} \int d^{3} x\left\langle T_{\mu \nu}(x) T_{\mu v}(0)\right\rangle .
$$

One can easily show that the integral in Eq. (3.11) for $1 \leq i<j \leq 3$ has an ultraviolet divergence in the continuum theory. In the measurement of Eq. (3.11) on the lattice, therefore, one has to take an appropriate regularization to remove the divergence. In this study, we simply regard that this regularization can be acheived by subtracting the vacuum part of the fluctuations,

$$
\left\langle T_{\mu \nu}^{2}\right\rangle_{\mathrm{reg}}=\left\langle T_{\mu \nu}^{2}\right\rangle_{T \neq 0}-\left\langle T_{\mu \nu}^{2}\right\rangle_{T=0}
$$

\section{Numerical results}

We performed lattice simulations for SU(3) pure gauge theory with a standard Wilson gauge action. Gauge configurations are updated by heatbath and overrelaxation algorithms. In Table 1 we list lattice parameters used in this work. The simulations are performed on four isotropic lattices to investigate the lattice spacing and volume dependence. The numbers of temporal lattice sites $N_{\tau}$ correspond to $T \sim 0.5 T_{c}-2.5 T_{c}$. The energy-momentum tensor in Euclidean space used in this work is given by $T_{\mu v}=2 \operatorname{Tr}\left[F_{\mu \rho} F_{v \rho}-\frac{1}{4} \delta_{\mu v} F_{\rho \sigma} F_{\rho \sigma}\right]$ where the energy-momentum tensor is constructed to be traceless, $T_{\mu \mu}=0$. For the definition of the field strength on the lattice, we have chosen the clover operator. For each parameter, hundreds of thousands of configurarions have been 


\begin{tabular}{cccccc}
\hline & $\beta=6 / g^{2}$ & $N_{\sigma}^{3}$ & $N_{\tau}$ & $a[\mathrm{fm}]$ & $L[\mathrm{fm}]$ \\
\hline case 1 & 6.499 & $32^{3}$ & $6,8,12,32$ & 0.049 & 1.6 \\
\hline case 2 & 6.205 & $32^{3}$ & $4,6,8,32$ & 0.074 & 2.4 \\
\hline case 3 & 6.000 & $32^{3}$ & $4,6,8,16$ & 0.094 & 3.0 \\
\hline case 4 & 6.000 & $16^{3}$ & $4,6,8,16$ & 0.094 & 1.5 \\
\hline
\end{tabular}

Table 1: Simulation parameters. $N_{\sigma}$ and $N_{\tau}$ are the numbers of lattice sites in spatial and temporal directions, respectively. $a$ and $L$ denote the lattice spacing and spatial lattice size, respectively.

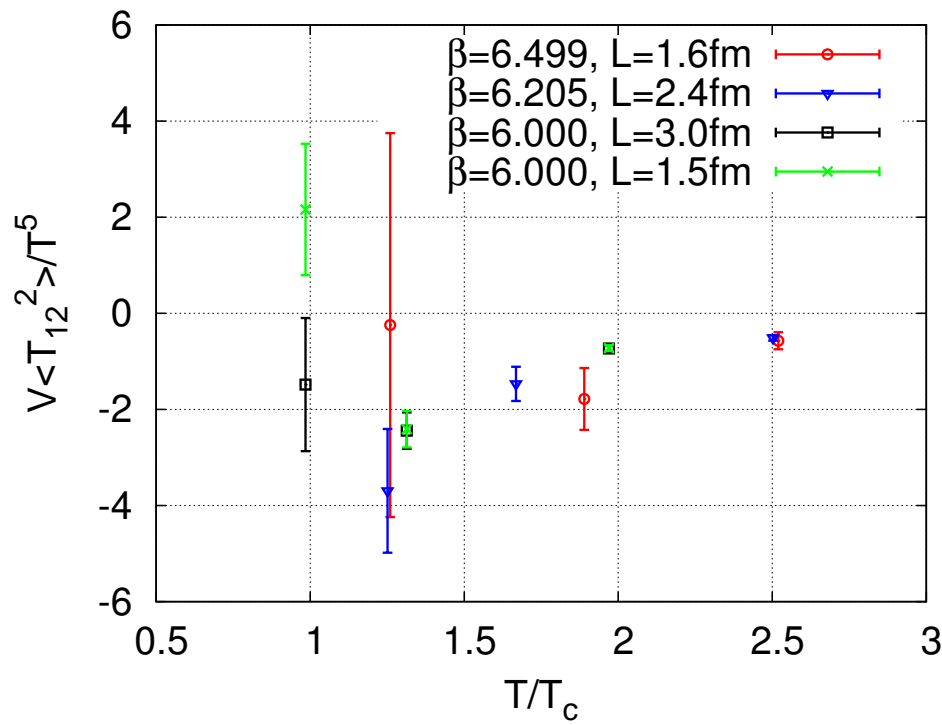

Figure 1: Fluctuations of an off-diagonal component of energy-momentum tensor per umit volume at finite temperature. The vaccum contribution is subtracted at each temperature. The fluctuations over $T_{c}$ have negative value and below $T_{c}$ are consistent with zero.

prepared. Statistical errors are estimated by the jackknife method with a bin size in the range $50-1000$.

As argued in Eq. 3.11), the fluctuation of energy-momentum tensor is an ultraviolet divergent quantity, and one must renormalize this vacuum contribution in the measurement. We regarded the fluctuations measured with largest $N_{\tau}$ for each set of configuration to be the value for the vacuum, and subtracted it following Eq. (3.12).

In Fig. 1, we show the $T$ dependence of fluctuations of the off-diagonal component of energy-

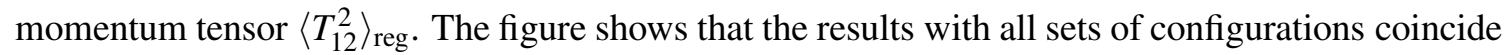
within the statistical error, which indicates that the lattice spacing dependence of this quantity is well suppressed. Moreover, by comparing the result with different $L$ for $\beta=6.0$ one finds that the spatial volume dependence is also small except for $T \simeq T_{c}$. The numerical result, however, shows that $\left\langle T_{12}^{2}\right\rangle_{\text {reg }}$ takes a negative value for all temperatures analyzed. Because $\left\langle T_{12}^{2}\right\rangle_{\text {reg }}$ is related to $\beta_{2}=\eta /\left(2 \tau_{\pi}\right)$ through Eq. (3.8), the result shows that one of the transport coefficients becomes 
negative, which, however, contradicts the stability of the hydrodynamic equations.

\section{Discussion}

In this work, we attempted to evaluate the second order transport coefficient $\beta_{2}$, i.e. a ratio between the relaxation time $\tau_{\pi}$ and the shear viscosity $\eta$ in SU(3) gauge theory by lattice simulations. Using Einstein principle (3.2) and the form of entropy in IS theory (3.4), the ratio can be related to statistical fluctuation of off-diagonal component of energy-momentum tensor in equilibrium through Eq. (3.8). Since the fluctuation of energy-momentum tensor has an ultraviolet divergence, we regularized the divergence by subtracting the vacuum fluctuation. We, however, found that with this regularization $\beta_{2}$ becomes negative. We also found that the wrong sign is not attributed to the lattice spacing or the spatial volume dependences, because the numerical result shows that effects of these quantities on observables measured in this study are small.

Negative value for $\beta_{2}$ may be due to the contribution from temperature-dependent divergence which cannot be renormalized by a naïve subtraction of vacuum contribution. This temperaturedependent contribution may arise from divergence at identical space-time point. Similar problem is known to occur for the vacuum expectation value of composite field composed of some local field operators. This analysis is in progress.

\section{Acknowledgement}

Lattice calculations have been performed with Blue gene in KEK and PACS-CS computer in Tsukuba. Y.K. is supported by Grant-in-Aid for Japan Society for the Promotion of Science.

\section{References}

[1] Quark-Gluon Plasma. New Discoveries at RHIC: Case for the Strongly Interacting Quark-Gluon Plasma. Nucl. Phys. A 750 (2005) 1.

[2] P. Arnold, G. D. Moore and L. G. Yaffe, JHEP 0011 (2000) 001 [arXiv:hep-ph/0010177].

[3] C. Eckart, Phys. Rev. 58 (1940) 919.

[4] L.D. Landau and E.M. Lifshitz, Fluid mechanics (Pergamon, New York, 1959).

[5] W. A. Hiscock and L. Lindblom, Annals Phys. 151 (1983) 466.

[6] W. Israel, Annals Phys. 100 (1976) 310; W. Israel and J. M. Stewart, Annals Phys. 118 (1979) 341.

[7] A. Muronga, Eur. Phys. J. ST 155 (2008) 107 [arXiv:0710.3280 [nucl-th]].

[8] S. Pratt, Phys. Rev. C 77 (2008) 024910 [arXiv:0711.3911 [nucl-th]].

[9] See also, a related work, T. Koide, E. Nakano and T. Kodama, Phys. Rev. Lett. 103 (2009) 052301 [arXiv:0901.3707 [hep-th]].

[10] A. Muronga, Phys. Rev. C 69 (2004) 034903 [arXiv:nucl-th/0309055].

[11] R. Baier, P. Romatschke, D. T. Son, A. O. Starinets and M. A. Stephanov, JHEP 0804 (2008) 100 [arXiv:0712.2451 [hep-th]].

[12] A. Einstein, Annals Phys. 33 (1910) 1277. 